\title{
Heurs et déboires des empreintes génétiques en médecine légale
}

Un long commentairedans Science [1], un éditorial [2] suivi d'un article très documenté [3]dans Nature, un communiqué commun du président du conseil de l'ordre des médecins et du bâtonnier de l'ordre des avocats et un article publié dans le Monde [4], pourquoi cet émoi récent à propos d'une technique de biologie moléculaire, celle des empreintes génétiques, qui a fait irruption en médecine légale depuis deux ans? En fait se posent deux problèmes tout à fait distincts, éthique et scientifique. Le problème éthique [4] vient de la crainte d'une intrusion dans la vie familiale et personnelle, dont les conséquences risquent d'être graves. Il fait l'objet de discussions constantes ; sans en sousestimer l'importance, c'est aux problèmes scientifiques, dont la gravité a été méconnue, que nous nous attacherons.

En 1985, à Londres, Alec Jeffreys décrivait pour la première fois l'obtention des «empreintes génétiques », spécifiques de chaque individu. Il appliquait la méthode de Southern à des régions d'ADN particulièrement polymorphes qu'il appela minisatellites. Rapidement la méthode fut codifiée: après attaque par des enzymes de restriction appropriées, on mettait en évidence des images complexes comportant un nombre assez élevé de bandes bien reproductibles. médecine/sciences, alors dans sa première année de parution, avait rapidement reconnu l'importance de cette découverte puisqu'elle la relatait quelques semaines après $\left(\mathrm{m} / \mathrm{s} n^{\circ} 6\right.$, vol. 1 , p. 333). Les jalons successifs étaient également portés à la connaissance de nos lecteurs : premières applications $\left(\mathrm{m} / \mathrm{s} n^{\circ} 3\right.$, vol. 2, p. 165) et commercialisation en Angleterre en $1987 \mathrm{~lm} / \mathrm{s}$ $n^{\circ} 7$, vol. 2, p. 401). Le parti que l'on pouvait en tirer pour identifier des individus, notamment en médecine légale, est d'emblée apparu considérable.

$\mathrm{m} / \mathrm{s} n^{\circ} 8$ vol. 5 , octobre 89
Deux progrès décisifs par rapport aux techniques classiques, en majorité immunologiques, justifiaient cet engouement: jusqu'à présent on pouvait atteindre une probabilité de l'ordre de $99 \%$ que deux sangs soient identiques. Suffisantes pour une exclusion ces méthodes ne permettaient pas une identification positive. Or les empreintes génétiques atteignent en principe des chiffres de probabilité défiant l'imagination : selon la nature et le nombre des sondes utilisées - et on peut toujours en ajouter d'autres - , on arrive à une chance sur 100 millions, des milliards... ou des milliards de milliards pour que deux individus différents aient la même empreinte, à l'exception des jumeaux monozygotes. Le deuxième progrès, particulièrement net depuis l'introduction de la méthode PCR (amplification de l'ADN), est la taille minime de l'échantillon requis. Il suffisait d'emblée de $1 \mu \mathrm{g}$ - et maintenant beaucoup moins - d'ADN, obtenu à partir d'une tache de sang, même ancienne, ou de sperme, et on peut se contenter aujourd'hui d'un seul cheveu ( $m / s n^{\circ} 7$, vol. 4, p. 457).

Une telle technique devait se frayer un chemin en médecine légale. En Angleterre, le premier problème abordé fut celui du « regroupement familial » des immigrants ; soupçonnant que des substitutions de personnes puissent se produire lorsqu'un parent - époux, enfant demande à rejoindre un immigrant déjà installé, on a appliqué la méthode avec succès pour obtenir des confirmations d'identité et même de parenté ( $m / s n^{\circ} 3$, vol. 2, p. 165). Un cas de cette nature a été mentionné en France [5] : après la mort par surdose d'un jeune homme, la mère, qui n'avait pu voir le corps, n'a pas reconnu, à la lecture du rapport d'autopsie, la description de son fils, et a mis en doute son identification. Elle demande donc que l'on confronte l'ADN extrait des tissus autopsiés au sien et à celui de son mari.

Mais bien entendu le terrain d'élection s'annonçait, celui des meurtres et des viols. Ce sont les compagnies privées qui se sont engouffrées dans la voie ainsi ouverte. Cela n'a pas été sans créer quelques complications : la méthode de Jeffreys a été confiée à Imperial Chemical Industries; des brevets ayant été pris, il s'ensuit que, ou bien on s'adresse à cette compagnie, ou bien la concurrence, si elle n'achète pas les brevets, doit mettre au point d'autres méthodes. En France, Appligène, une compagnie strasbourgeoise, a eu recours à une technique belge $\left(\mathrm{m} / \mathrm{s} n^{\circ} 6\right.$, vol. 3 , p. 370) ; les compagnies américaines, Lifecodes et Cellmark, ont adopté d'autres polymorphismes de restriction.

Introduite en 1987, la technique a été utilisée de plus en plus souvent, par exemple dans une centaine de cas aux États-Unis ; ses références scientifiques emportaient en général la conviction du tribunal et des jurés : "you can't argue with science ", disait un de ces derniers. Lorsque, au contraire, les résultats ont été mis en doute, le choc fut considérable. En 1987, dans un milieu hispanique, une femme et sa fille furent poignardées dans leur appartement du Bronx. Un voisin, soupçonné, avait une montre qui portait une petite tache de sang. Les techniciens de Lifecodes réussirent à en extraire $0,5 \mu \mathrm{g}$ d'ADN, auquel ils appliquèrent les trois sondes qu'ils utilisent habituellement ; ils conclurent que les bandes de la montre et celles de la femme coïncidaient, avec une chance sur 190000000 de non-identité. D'où condamnation inévitable du suspect. Pour la première fois, les avocats de la défense décidèrent de mettre en cause les résultats de l'analyse de l'ADN et de les faire discuter en une 
session judiciaire spéciale dite Frye hearing: il s'agit d'une jurisprudence datant de 1923 qui stipule qu'une méthodologie nouvelle ne peut être introduite en justice que s'il est avéré qu'elle est acceptée par la communauté scientifique, et notamment que des faux positifs sont pratiquement impossibles. La session eut lieu au début de 1989, et ne se passa pas comme prévu.

Un des avocats de la défense, Peter Neufeld, avait assisté récemment à une réunion sur l'emploi de l'ADN en médecine légale, réunissant biologistes moléculaires, magistrats, avocats et policiers. Parmi les biologistes se trouvait Eric Lander (Cambridge, MA, USA), future figure centrale du procès. Il réagit vivement quand le représentant de Lifecodes montra un autoradiogramme portant deux pistes, et dit que les bandes concordaient, malgré un décalage apparent, parce que l'une des pistes avait migré plus vite que l'autre ; aucun standard n'était inclus dans l'expérience. Après cette présentation, et une autre venant de Cellmark, concurrent de Lifecodes, il devenait clair que les précautions considérées comme élémentaires en recherche n'étaient pas respectées. Au moment du procès, un expert de l'accusation, Richard Roberts, proposa à Lander et aux autres experts commis dans l'affaire de tenir ensemble une réunion à caractère scientifique, en dehors de toute présence de légiste. Ils parvinrent, semble-t-il, assez facilement à des conclusions communes, mettant en cause la méthode employée et les conduisant, experts de l'accusation et de la défense confondus, à récuser les conclusions de l'analyse de l'ADN, par une démarche sans précédent dans les annales judiciaires. Dans son article de Nature, Lander [3] développe les critiques en détail. Nous ne pouvons ici les relater toutes, mais seulement donner une idée des approximations auxquelles ont eu recours les analystes dans ce cas. Les résultats obtenus avec les trois sondes utilisées soulevaient plusieurs points litigieux.

a. Le nombre de bandes : le rapport Lifecodes décrit trois bandes pour la sonde DXYS14 dans chacune des trois pistes, «montre », mère et fille. pas à ce que l'on voit sur l'autoradiogramme; si la piste de la mère porte effectivement trois bandes, on en dénombre cinq sur celle de la montre et une seule sur celle de la fille. Une contamination du sang par des bactéries, possible mais non prouvée, était alléguée pour expliquer la présence des bandes supplémentaires qu'on a négligées.

b. La position des bandes : pour les deux autres sondes, la correspondance exacte mère-montre est loin d'être évidente. Lifecodes déclare se servir d'un ordinateur pour établir la concordance des bandes, puis admet utiliser en pratique l'évaluation visuelle; or beaucoup d'allèles ont une position très voisine, et même ainsi des décalages semblaient évidents.

c. Une sonde supplémentaire donnant une bande spécifique du chromosome Y a pour objet d'établir le sexe des sujets en cause. La bande marqueur était absente de la montre comme de la mère et de la fille, mais aussi du témoin utilisé dans l'expérience... dont il a été impossible de retrouver de qui il émanait, homme ou femme! Aucun marqueur positif du chromosome Y n'était donc fourni.

d. Dégradation de l'ADN : ce risque, que bien entendu on ne peut imputer à ceux qui pratiquent l'analyse, est le facteur d'erreur le plus important de la technique. Or l'ADN provenant du sang de la montre était manifestement dégradé, et la plupart des allèles de la population hispanique, qui dépassent $10 \mathrm{~kb}$, n'apparaissaient pas. La compagnie a bien fourni un autoradiogramme supposé montrer une bande de $23 \mathrm{~kb}$, mais l'avocat Neufeld, devenu un expert en lecture de Southern blots, fit remarquer qu'on avait mal lu la position des marqueurs, et qu'aucune bande ne dépassait $10 \mathrm{~kb}$.

e. Une dernière cause d'erreur possible, portant cette fois sur les probabilités d'unicité des empreintes, dérive de la génétique des populations. La population hispanique et ce trait ne lui est nullement réservé - est loin d'être homogène, et certains marqueurs peuvent se concentrer dans des familles données : on peut ainsi calculer des probabilités d'identité très inférieures à la réalité si l'on postule l'homogénéité de la population.

Les débats se terminèrent le 26 mai 1989, 15 semaines après leur début ; la décision attendue ne portera que sur la recevabilité du témoignage de l'ADN, et le procès de José Castro le crime date du 5 février 1987 n'aura lieu qu'ensuite.

Une conséquence importante de ces débats est que des jugements émis antérieurement sur le témoignage de l'ADN risquent d'être révisés. Lander en décrit quatre exemples, nous ne relaterons que le dernier : en février 1988 , une femme amena sa voiture dans un garage pour réparation. Le mécanicien affirma avoir trouvé un bébé mort sur le siège arrière. Malgré les dénégations de la femme (corroborées par son médecin) qui niait avoir été enceinte, Cellmark conclut qu'elle était bien la mère d'après l'analyse de l'ADN ; en effet, sur huit bandes caractéristiques, elle en partageait quatre avec l'enfant, or on sait qu'un enfant hérite de la moitié des bandes caractéristiques de chacun de ses parents. La femme ne fut cependant pas inculpée d'infanticide car l'enfant était mort-né. En octobre de la même année, elle accouchait d'un enfant à terme, conçu, d'après les échographies, en janvier, donc peu avant la naissance de l'enfant qui lui était attribué... Cellmark a entrepris d'analyser à nouveau l'ADN du bébé mort, les conclusions de la première analyse étant probablement erronées. Il reste maintenant à tirer les enseignements de ces débats pour l'avenir. Le plus fâcheux serait qu'ils jettent le discrédit sur la méthode au point que personne n'oserait plus la préconiser. Ce qu'il faut, c'est fixer des règles précises pour la technologie, et peut-être pour des habilitations. L'exemple du diagnostic prénatal en France peut être évoqué : il connaît une large diffusion, et pourtant il est fiable, seuls les laboratoires reconnus comme possédant, et appliquant, la technologie adéquate étant autorisés à le pratiquer. Il ne s'agit pas d'interdire à des laboratoires privés, dont le profit est le seul moteur, de mettre en œuvre une technique, mais de veiller à ce qu'ils ne négligent pas les précautions nécessaires à seule fin d'abaisser les coûts. Ce domaine souffre aussi, par rapport à notre référence, le diagnostic prénatal, 
d'un handicap considérable, qui n'a peut-être pas été souligné. En prénatal, les méthodes sont publiées, on les reproduit dans la plupart des laboratoires ou on va en faire l'apprentissage chez un expert. Pour les empreintes génétiques, nous l'avons dit au début, des brevets sont pris, chaque laboratoire doit mettre au point ses méthodes sans pouvoir bénéficier de l'expérience des autres. Nulle part, dans ce que nous avons lu, ne figure la suggestion d'une collaboration technique internationale*. Aux États-Unis, comme il est habituel, on ne prend en considération que le territoire national, et on invite l'Académie des sciences à prendre l'affaire en main - ou peut-être le FBI. Aucune allusion n'est faite à l'expérience que pourraient avoir les Britanniques, pionniers de la méthode. L'éditorial non signé de

* A la notable exception près du communiqué de MM. Louis René et Philippe Lafarge, Bulletin du conseil de l'ordre des médecins, juillet-août-septembre 1989, $n^{\circ} 51$.
Nature - journal international mais dont la base principale reste Londres - ne l'évoque pas non plus [2]. Chaque pays risque donc d'avoir à faire sa propre expérience, avec les tâtonnements que cela implique.

En conclusion, il ne faut pas demander aux empreintes génétiques plus qu'elles ne peuvent donner. Notamment, les résultats provenant d'un ADN dégradé risquent d'être ininterprétables. Mais les difficultés ne sont certes pas propres à cette technique en médecine légale. Combien n'avons-nous pas vu de ces batailles d'experts, les uns affirmant catégoriquement ce que les autres récusent avec une égale énergie. La différence, c'est qu'une méthode aussi élaborée que l'analyse de l'ADN devrait aboutir à des conclusions indiscutables. Il faudra donc établir des règles, aussi rigoureuses que celles qu'on applique aux diagnostics médicaux. L'intérêt général voudrait aussi que l'on ne puisse pas breveter les meilleures techniques en médecine légale, car cela oblige la concurrence à recourir à des méthodes éventuellement moins fiables. Mais ceci est une autre histoire...

\section{Jean-Claude Dreyfus}

\section{RÉFÉRENCES}

1. Lewin R. DNA typing on the witness stand. Science $1989 ; 244$ : 1033-5.

2. Editorial (non signé). Fingerprinting trials. Nature 1989 ; 339 : 491-2.

3. Lander ES. DNA fingerprinting on trial. Nature 1989 ; 339 : 501-5.

4. René L, Lafarge P. Génétique : empreintes sans bavures. Le Monde, 5 juillet 1989.

5. Nau JY. La technique des empreintes génétiques est mise en œuvre pour identifier un cadavre. Le Monde, 31 mars 1989.

DERNIĖRE MINUTE : la décision du juge, prise le 14 août 1989. fut de rejeter les conclusions de I'analyse de I'ADN dans le procès Castro; il invite de plus les cours de justice à une extrême prudence dans les cas à venir (rapporté par Norman C. Science 1989 ; 245 : 699). 